\title{
Peroxiredoxin 6 overexpression regulates adriamycin-induced myocardial injury, oxidative stress and immune response in rats
}

\author{
Jianshu Guo ${ }^{1 *}$, Weiping Cao ${ }^{2 *}$, Chi Chen ${ }^{1}$, Xiaohan Chen $^{1}$ \\ ${ }^{1}$ Department of Geriatric Medicine, Sichuan Academy of Medical Sciences \& Sichuan Provincial People's Hospital, School of Medicine, University \\ of Electronic Science \& Technology, Chengdu, China; ${ }^{2}$ Department of Cardiology, People's Hospital of Leshan, Leshan, China \\ Contributions: (I) Conception and design: J Guo, W Cao; (II) Administrative support: X Chen; (III) Provision of study materials or patients: C Chen; \\ (IV) Collection and assembly of data: J Guo; (V) Data analysis and interpretation: W Cao; (VI) Manuscript writing: All authors; (VII) Final approval \\ of manuscript: All authors. \\ "These authors contributed equally to this work. \\ Correspondence to: Chi Chen; Xiaohan Chen. Department of Geriatric Medicine, Sichuan Academy of Medical Sciences \& Sichuan Provincial People's \\ Hospital, School of Medicine, University of Electronic Science \& Technology, No. 32 West Second Section, First Ring Road, Chengdu, China. \\ Email: guojianshu1@126.com; cher19@163.com.
}

\begin{abstract}
Background: Adriamycin is an anthracycline drug used to treat a variety of tumors. Adriamycin has a much stronger affinity for myocardial tissue than other body tissues. Cancer patients treated with adriamycin are prone to toxic damage to heart tissue. Peroxiredoxin 6 (PRDX6) is a novel antioxidant enzyme in metabolic diseases, the aim of this study was to investigate the role of PRDX6 in myocardial injury.

Methods: Sixty male specific-pathogen-free Wistar rats were enrolled and divided equally into the control group (control), the Adriamycin group, the Adriamycin + empty vector lentivirus (Adriamycin $+\mathrm{LV}$ ) group, and the Adriamycin + Peroxiredoxin 6 overexpression (Adriamycin + PRDX6) group. Western blot, reverse transcription-polymerase chain reaction (PCR), enzyme-linked immunosorbent assay, hematoxylin and eosin staining (HE) and immunohistochemistry were used in this research.

Results: The myocardial tissues of the Adriamycin group had significantly lower expression levels of PRDX6 and PRDX6 mRNA than those of the control group, and the myocardial tissues of the Adriamycin + PRDX6 rats had significantly higher expression levels of PRDX6 and PRDX6 mRNA than those of the Adriamycin + LV group. Serum creatine kinase isoenzyme (CK-MB), myoglobin (Mb), cardiac troponin I (cTnI), myocardial injury, positive rate of caspase-3, B-cell lymphoma 2 (Bcl-2)/Bcl-2-associated X protein (Bax) levels, malondialdehyde (MDA), lactate dehydrogenase (LDH), IL-1 $\beta$, IL-6, inducible nitric oxide synthase (iNOS), and IL-4 proteins in the adriamycin-induced rats were significantly higher than those in the control group, while superoxide dismutase (SOD) activity was significantly lower than that in the control group. PRDX6 overexpression reversed the above results.

Conclusions: PRDX6 overexpression can alleviate adriamycin-induced myocardial injury in rats, which may be related to oxidative stress regulation and the levels of inflammatory factors.
\end{abstract}

Keywords: Adriamycin; myocardial injury; peroxiredoxin 6 (PRDX6); oxidative stress; inflammatory factor

Submitted Aug 28, 2020. Accepted for publication Oct 09, 2020.

doi: $10.21037 /$ atm-20-6598

View this article at: http://dx.doi.org/10.21037/atm-20-6598

\section{Introduction}

Doxorubicin (also known as adriamycin) is an anthracycline drug that is used in the treatment of various tumors. Doxorubicin acts on the DNA chemical structure of cells to influence their physiological activities, and demonstrates a broader anti-tumor effect than other anthracycline drugs. Doxorubicin has been used in combination with targeted therapy and chemotherapy drugs as a first-line clinical 
treatment for solid and hematological tumors $(1,2)$.

Despite showing good clinical effects as an antitumor treatment, doxorubicin use is often accompanied by toxicity and side effects, such as platelet reduction and leukopenia. Doxorubicin has a much stronger affinity for myocardial tissue than for other body tissues. Therefore, cancer patients treated with doxorubicin are prone to toxic damage to cardiac tissue, which increases the amount of free radicals in the myocardial tissue, inhibits nucleic acid and protein synthesis, and hinders energy metabolism, causing irreversible damage to the heart. Severe cases may even result in heart failure or death $(3,4)$.

Peroxiredoxins are a class of selenium-independent peroxidases with antioxidant activity, which are widely distributed in mammals. Peroxiredoxin 6 (PRDX6), one of the members of this family $(5,6)$, is a 1 -Gys enzyme comprising $224 \mathrm{AA}$ and electrons provided by glutathione. PRDX6 is expressed in various tissues such as the epidermis, apical area, and respiratory epithelium. PRDX6 not only has the function of a glutathione peroxidase, but it also has the activity of phospholipase A2, can improve the lipid peroxidation reaction, and has an antioxidant effect (7). Studies $(8,9)$ have shown that PRDX6 can reduce the severity of corneal damage caused by ultraviolet radiation, oxidative stress, inflammation, and neovascularization. Moreover, PRDX6 can also inhibit airway inflammation induced by lipopolysaccharide (10). There has been a few reports of the effect of PRDX6 on doxorubicin-induced myocardial injury recently. Therefore, to our best knowledge, this study was the first time to explore the effect of PRDX6 on doxorubicininduced myocardial injury in rats by constructing a PRDX6 overexpression vector using lentiviral plasmids, and to provide a reference for the design of drugs to alleviate injury caused by Adriamycin-induced cardiotoxicity.

We present the following article in accordance with the ARRIVE reporting checklist (available at ttp://dx.doi. org/10.21037/atm-20-6598).

\section{Methods}

\section{Materials and instruments}

\section{Animals}

Specific-pathogen-free (SPF) male Wistar rats (10 weeks old, 280-300 g) were obtained from the Southern Medical University [SCXK (Guangdong) 2016-0041]. All experiments are conducted according to the $3 \mathrm{R}$ principle for animal experiments and approved by the ethics committee.

\section{Reagents}

Adriamycin (abs81723350 mg, Abison, Shanghai Biotechnology, China); TRIzol column total RNA purification kit (MK020001, Hangzhou Lianke Biotechnology, China); Revertaid First Strand cDNA Synthesis Kit (K1622, Shanghai Yisheng Biotechnology, China); SYBR Green Realtime PCR Master Mix (QPK-201, Shanghai Huzhen Industrial); protein extraction kit (SD-001/ SN-002, Invent, USA); rabbit polyclonal PRDX6 Antibody, mouse anti-B-cell lymphoma 2 (Bcl-2) and mouse anti-Bcl-2associated X protein (Bax) antibody (ATA35878, ATA25286, ATA25316, Wuhan Yipu Biotechnology, China); rabbit antiiNOS antibody and mouse anti-IL-4 antibody (FNab04325, Fnab04279, Wuhan Fine Biotechnology, China); Rabbit Anti-Caspase-3 antibody (BW11044, Shanghai Qiming Biotechnology Co., Ltd.); creatine kinase isoenzyme (CK$\mathrm{MB}$ ) enzyme-linked immunosorbent assay (ELISA) kit (E-EL-R1327km, Shanghai Hengfei Biotechnology, China); muscle Red protein $(\mathrm{Mb})$ and inducible nitric oxide synthase (iNOS), ELISA kit (YS05614B, YS03982B, Shanghai Yaji Biotechnology, China); cardiac troponin I (cTnI) ELISA kit (GD-DX1962, Shanghai Ancient Do Biotechnology, China); malondialdehyde (MDA), lactate dehydrogenase (LDH), superoxide dismutase (SOD), and interleukin (IL)-4 ELISA kits (MM-0385R1, MM-0605R1, MM-0386R1, MM0191R1, Wuhan Yipu Biotechnology, China); IL-1 $\beta$ and IL-6 ELISA kits (YE01823, YE0013, Shanghai Yuanmu Biological Technology, China).

\section{Instruments}

A high-speed refrigerated centrifuge (Avanti JXN-26) was purchased fromBeckman Coulter, a fluorescence microscope (DMi8-electric) was purchased from, Leica, a real-time fluorescent quantitative polymerase chain reaction (PCR) instrument (LightCycler 480 II) was purchased from Roche, a multifunctional microplate reader (SpectraMax iD3) was purchased from US Molecular Devices, and an ultra-low temperature refrigerator (CryoCube F570) was purchased from Eppendorf.

\section{Experimental metbods}

The PRDX6 overexpression lentiviral vector was constructed and packaged by Shanghai GenePharma Co., Ltd. (Shanghai, China) and verified by members of the research team. Sixty male SPF Wistar rats were enrolled and divided into four groups ( $\mathrm{n}=15$ in each group) as follows: the control group (Control), the Adriamycin group, 
the Adriamycin + empty vector lentivirus (Adriamycin + LV) group, and the Adriamycin + PRDX6 overexpression lentivirus vector (Adriamycin + PRDX6) group. The rats in the Adriamycin + LV and Adriamycin + PRDX6 groups were injected with empty lentiviral plasmid and PRDX6 overexpression lentiviral vector $\left(5 \times 10^{7} \mathrm{TU} / \mathrm{head}\right)$, respectively, via the tail vein. The rats in the control and Adriamycin group were injected with the same volume of normal saline via the tail vein (once a day for 3 days). On day 4 after the last injection, all the rats in the three experimental groups were intraperitoneally injected with $12.5 \mathrm{mg} / \mathrm{kg}$ Adriamycin (4), while all the rats in the control group were intraperitoneally injected with the same volume of normal saline (once a day for 7 days).

\section{Sample collection}

The rats were euthanized with $3 \%$ sodium barbital 24 hours after the last injection of Adriamycin, and 1,000 IU/kg heparin was injected intraperitoneally. Following that, $2 \mathrm{~mL}$ of blood was drawn out of the heart and stored in a sterile centrifuge tube at $-20{ }^{\circ} \mathrm{C}$ for future use. The heart tissue was quickly removed and placed in the potassium hydride (KH) solution at $4{ }^{\circ} \mathrm{C}$; the excess tissue around the rat heart was trimmed, and residual blood in the heart was removed. Then, part of the heart tissue was fixed in 4\% formaldehyde neutral solution for hematoxylin and eosin (HE) staining and caspase-3 immunohistochemistry detection. The rest of the heart tissue was stored at $-80{ }^{\circ} \mathrm{C}$ for future use, including the detection of myocardial tissue oxidative stress factors and inflammatory factors, and western blot analysis.

\section{Detection of serum beart damage markers}

The blood drawn out of the rat heart was centrifuged at 3,500 $\mathrm{r} / \mathrm{min}$ for $10 \mathrm{~min}$. The supernatant was then taken, and the serum levels of CK-MB, Mb, and cTnI were detected by performing ELISA in strict accordance with the manufacturer's instructions. The experiment was performed in triplicate.

\section{Detection of oxidative stress and inflammatory factors in myocardial tissue}

Lysates from tissues and cells were added with protease inhibitor to $0.5 \mathrm{~g}$ of myocardial tissue. These tissues were ground into tissue homogenate, which was then centrifuged at 3,000 r/min for $20 \mathrm{~min}$. After that, the levels of SOD, MDA, LDH, IL-1 $\beta$, IL-6, iNOS, and IL-4 in the supernatant were detected by ELISA. Detection was performed in strict accordance with the instructions of the kit and repeated 3 times.

\section{HE staining of myocardial tissue}

Fixed myocardial tissue was embedded in conventional paraffin, cut to a thickness of $4 \mu \mathrm{m}$, and then conventionally deparaffinized, hydrated, and stained with HE. Finally, the tissue was mounted with neutral gum and the pathological changes of the myocardial tissue were observed (11). The experiment was repeated 3 times.

\section{Myocardial tissue immunobistochemistry}

Fixed myocardial tissue was embedded in conventional paraffin, cut to a thickness of $4 \mu \mathrm{m}$, and then conventionally deparaffinized and hydrated. Next, the tissue was incubated with $3 \% \mathrm{H}_{2} \mathrm{O}_{2}$ at room temperature for $10 \mathrm{~min}$. After that, the tissue was washed with phosphate-buffered saline (PBS), sealed with goat serum, and then incubated with Caspase-3 antibody (1:300) overnight at $4{ }^{\circ} \mathrm{C}$. Subsequently, the secondary antibody was added to react at $37{ }^{\circ} \mathrm{C}$ for $15 \mathrm{~min}$. The tissue was next washed with PBS, stained with $0.05 \% 3,3$ 'diaminobenzidine (DAB) for $1 \mathrm{~min}$ for color development, and counterstained with hematoxylin for 5 min. Finally, after dehydrating and permeating, the sheet was sealed with neutral gum. The number of cells stained positive for caspase-3 was observed under a microscope.

\section{Western blot}

Total protein was extracted from rat myocardial tissue with a protein extraction kit, and the Bradford assay was performed for protein quantification. After sodium dodecyl sulphate-polyacrylamide gel electrophoresis (SDS-PAGE), the protein was transferred to a polyvinylidene difluoride (PVDF) membrane, sealed for $2 \mathrm{~h}$, and then incubated with PRDX6, iNOS, IL-4, Bax, Bcl-2, $\beta$-actin primary antibody (1:500) overnight at $4{ }^{\circ} \mathrm{C}$. After that, the membrane was rinsed with TBST (tris-buffered saline + Tween 20) for $40 \mathrm{~min}$, and incubated with horseradish peroxidaselabeled secondary antibody $(1: 500)$ at $37^{\circ} \mathrm{C}$ for $1 \mathrm{~h}$. Then, the membrane was rinsed with TBST for $40 \mathrm{~min}$, and developed with electrochemiluminescence (ECL). Finally, Image Lab software (Bio-rad, USA) was used to analyze the relative gray value of the target protein in each group of 

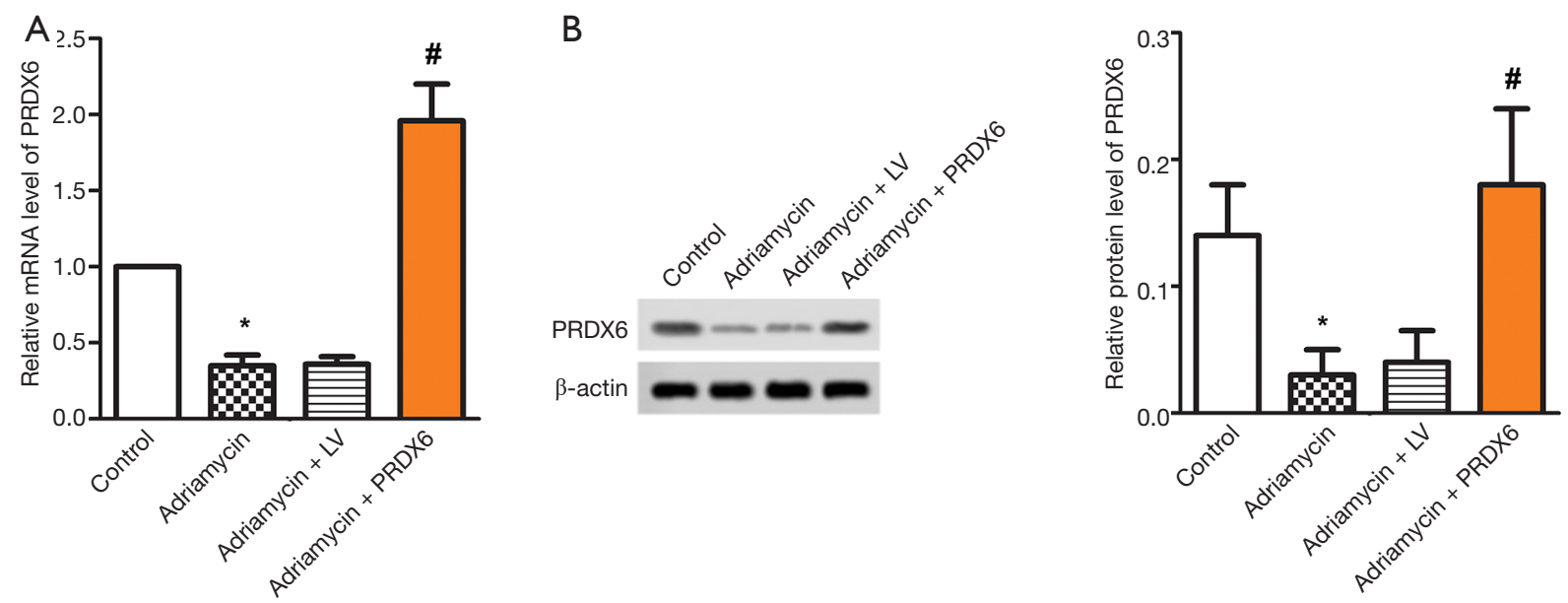

Figure 1 Validation of the PRDX6 overexpression rat model. (A) Histogram of the relative expression level of PRDX6 mRNA in the myocardial tissue of each group; (B) histogram of the relative level of PRDX6 protein in the myocardial tissue of each group. *, $\mathrm{P}<0.05$ compared with Control group; ${ }^{\#}, \mathrm{P}<0.05$ compared with Adriamycin $+\mathrm{LV}$ group.

cells. The experiment was repeated three times.

\section{Reverse transcription-PCR to detect PRDX6 expression level}

A TRIzol column total RNA purification kit was used to extract total RNA from rat myocardial tissue, the Revertaid First Strand cDNA Synthesis Kit was used to synthesize cDNA, and SYBR Green Real-Time PCR Master Mix was used to detect the expression level of PRDX6 in each group of cells. The cycling protocol was: $95^{\circ} \mathrm{C} 5 \mathrm{~min}, 95^{\circ} \mathrm{C} 30 \mathrm{~s}, 72{ }^{\circ} \mathrm{C} 30 \mathrm{~s}, 72{ }^{\circ} \mathrm{C}$ $10 \mathrm{~min}$, for 28 cycles. The primer sequences (Shanghai Shenggong) used were as follows: PRDX6, forward primer 5'-ATCCTCTACCCAGCCACC-3', reverse primer 5'-CCACGCCACAATCTTTCT-3'; $\beta$-actin, forward primer 5'-CTGTCTGGCGGCACCACCAT-3', reverse primer 5'-GCAACTAAGTCATAGTCCGC -3'. Quantitative PCR (qPCR) and the $2^{-\Delta \Delta \mathrm{Ct}}$ method were used to analyze gene expression.

\section{Statistical analysis}

Data were expressed as mean \pm standard deviation, and the t-test was used to analyze differences between two groups. Statistical analyses were conducted with SPSS 22.0 software (IBM, USA), and the histogram was created with GraphPad Prism (GraphPad, USA). $\mathrm{P}<0.05$ was set as the significant level.

\section{Results}

\section{Verification of the PRDX6 overexpression rat model}

The expression levels of PRDX6 mRNA and PRDX6 in the rat myocardial tissue were detected by RT-PCR and western blot. The Adriamycin group had significantly lower expression levels of PRDX6 mRNA and PRDX6 than the control group $(\mathrm{P}<0.05)$, while the expression levels of PRDX6 mRNA and PRDX6 in the Adriamycin + PRDX6 group were significantly higher than those in the Adriamycin $+\mathrm{LV}$ group $(\mathrm{P}<0.05)$. These results indicated that the PRDX6 overexpression rat model had been constructed successfully (Figure 1).

\section{The effects of PRDX6 overexpression on the levels of serum central injury markers}

The serum levels of CK-MB, $\mathrm{Mb}$, and $\mathrm{cTnI}$ in each group of rats were detected by ELISA. The results showed that the levels of serum CK-MB, Mb, and cTnI in the Adriamycin group were significantly higher than those in the control group $(\mathrm{P}<0.05)$, while the serum levels of $\mathrm{CK}-\mathrm{MB}, \mathrm{Mb}$, and cTnI in the Adriamycin + PRDX6 group were significantly lower than those in the Adriamycin $+\mathrm{LV}$ group $(\mathrm{P}<0.05)$. These results suggested that PRDX6 overexpression reduced the levels of CK-MB, $\mathrm{Mb}$, and $\mathrm{cTnI}$ in the serum of rats with myocardial injury caused by doxorubicin (Figure 2). 

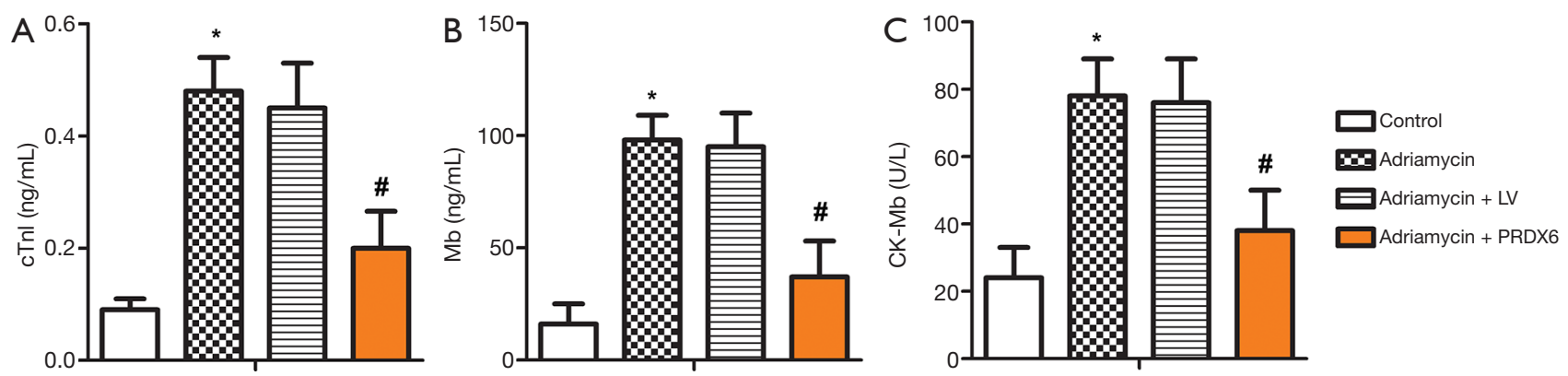

Figure 2 The effects of PRDX6 overexpression on the levels of serum central injury markers. (A) Histogram of serum cTnI levels in each group of rats; (B) histogram of the serum Mb levels in each group of rats; (C) histogram of serum CK-MB levels in each group of rats. *, $\mathrm{P}<0.05$ compared with control group;, $\mathrm{P}<0.05$ compared with Adriamycin $+\mathrm{LV}$ group.

\section{The effects of PRDX6 overexpression on myocardial pathological changes and apoptosis-related protein levels}

HE staining was performed to observe the pathological changes of the myocardial tissue of rats in each group. The results showed that the myocardial tissue cells of the Adriamycin and Adriamycin + LV rats were disordered, with severe cell necrosis; however, the myocardial tissue cells of the control and Adriamycin + PRDX6 groups were neatly arranged and without obvious cell necrosis. This phenomenon indicated that PRDX6 overexpression alleviated the severity of the myocardial injury caused by doxorubicin (Figure 3A). The expression level of Caspase-3 and the protein level of $\mathrm{Bax} / \mathrm{Bcl}-2$ in the rat myocardial tissue were detected by performing immunohistochemistry and western blot. The results showed that the positive rate of caspase-3 and the Bax/Bcl-2 level in the Adriamycin group were significantly higher than those in the control group $(\mathrm{P}<0.05)$. Myocardial tissue from the Adriamycin + PRDX6 group had a significantly lower positive rate of caspase- 3 and level of $\mathrm{Bax} / \mathrm{Bcl}-2$ than myocardial tissue from the Adriamycin $+\mathrm{LV}$ group $(\mathrm{P}<0.05)$. These results indicated that $\mathrm{PRDX} 6$ overexpression reduced the positive rate of caspase- 3 and the level of $\mathrm{Bax} / \mathrm{Bcl}-2$ in the myocardial tissue of rats with doxorubicin-induced myocardial injury (Figure 3B,C,D).

\section{The effects of PRDX6 overexpression on the levels of myocardial oxidative stress markers}

ELISA was used to detect the levels of SOD, MDA, and $\mathrm{LDH}$ in the myocardial tissues of rats in each group. The results showed that the Adriamycin group had significantly higher levels of MDA and LDH than the control group
$(\mathrm{P}<0.05)$, but the activity of SOD was significantly lower than that in the control group $(\mathrm{P}<0.05)$. The levels of MDA and LDH in the Adriamycin + PRDX6 group were significantly lower than those in the Adriamycin $+\mathrm{LV}$ group $(\mathrm{P}<0.05)$; however, SOD activity showed the opposite result $(\mathrm{P}<0.05)$. These results indicated that PRDX6 overexpression reduced the levels of MDA and $\mathrm{LDH}$ in rats with doxorubicin-induced myocardial injury while increasing SOD activity (Figure 4).

\section{The effects of PRDX6 overexpression on the levels of myocardial inflammatory factors}

The levels of IL-1 $\beta$, IL- 6 , iNOS, and IL-4, and the protein levels of iNOS and IL-4 in the myocardial tissue of each group of rats were detected using ELISA and western blot. The results showed that the levels of IL-1 $1 \beta$, IL-6, iNOS, and IL-4, as well as the iNOS and IL-4 protein levels, in the Adriamycin rats were significantly higher than those in the control group $(\mathrm{P}<0.05)$. Meanwhile, the levels of IL-1 $\beta$, IL-6, and iNOS, and the iNOS protein level, in the Adriamycin + PRDX6 rats were significantly lower than those in the Adriamycin $+\mathrm{LV}$ group $(\mathrm{P}<0.05)$, while the IL-4 and IL-4 protein levels were significantly higher $(\mathrm{P}<0.05)$. These results suggested that PRDX6 overexpression reduced the levels of IL-1 $\beta$, IL-6, iNOS, and iNOS protein in the myocardial tissue of rats with myocardial injury caused by doxorubicin, while increasing the protein level of IL-4 (Figure 5).

\section{Discussion}

Doxorubicin is an anti-tumor drug that can be used to treat a broad range of malignancies. However, the clinical application of doxorubicin is greatly limited by its strong, 

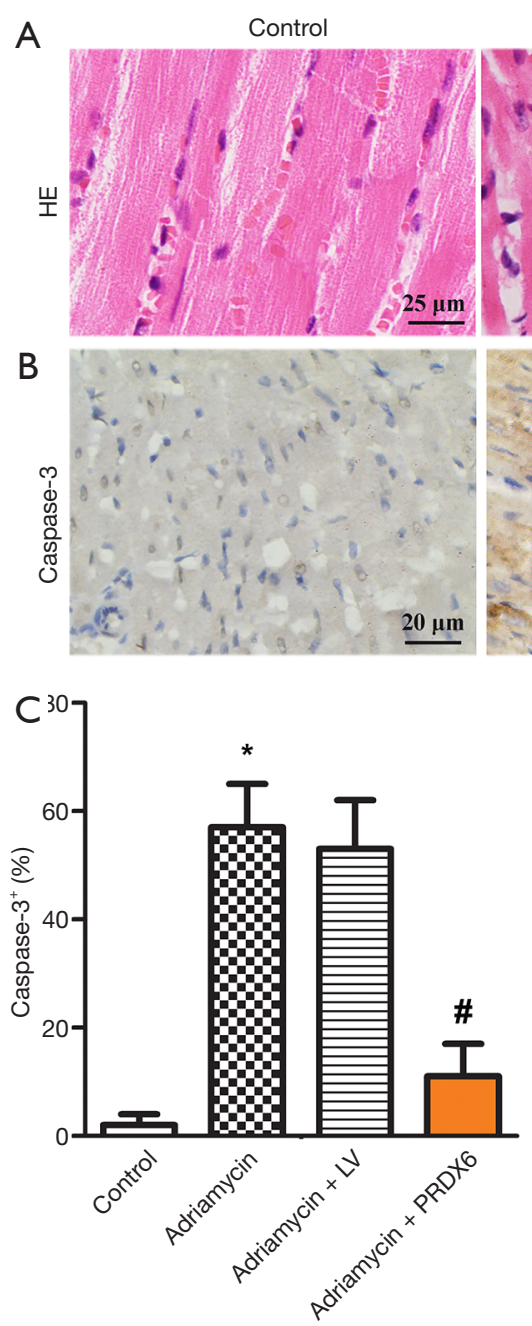

Adriamycin
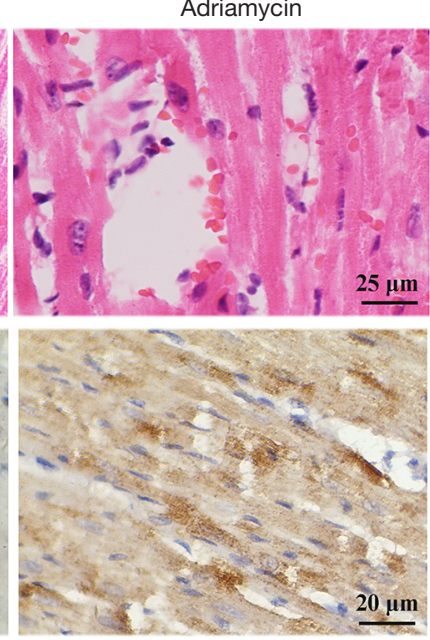

D

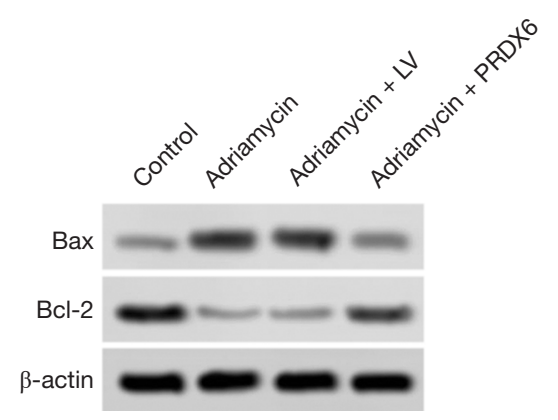

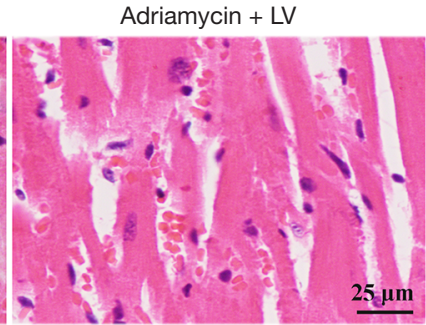
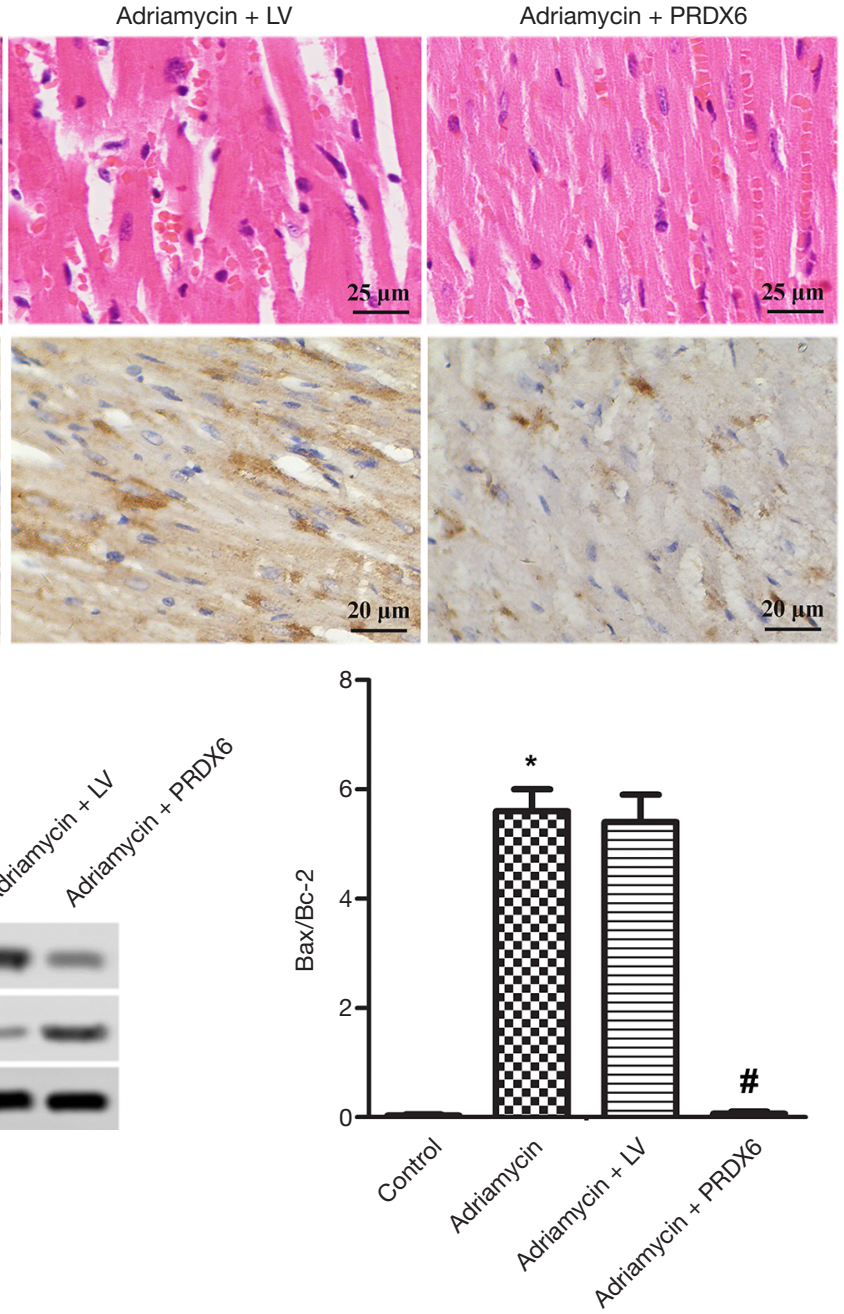

Figure 3 The effects of PRDX6 overexpression on myocardial pathological changes and apoptosis-related protein levels. (A) The results from hematoxylin and eosin staining $(\times 200)$ and (B) caspase- 3 immunohistochemistry $(\times 200)$ of myocardial tissue from each group of rats; (C) histogram of the number of caspase-3-positive cells in myocardial tissue from each groups of rats; (D) Gel imaging results showing the protein levels of Bax/Bcl-2 in myocardial tissue from each group, and the histogram of the protein levels of Bax/Bcl-2 in myocardial tissue of rats in each group. * $\mathrm{P}<0.05$ compared with control group; ${ }^{*}, \mathrm{P}<0.05$ compared with Adriamycin $+\mathrm{LV}$ group.

dose-dependent toxic effects on the heart of patients (12). The toxic damage to heart tissue caused by doxorubicin is related to cardiomyocyte apoptosis, cell mitochondrial damage, calcium overload, and superoxidation of cardiovascular endothelial cells $(13,14)$.

In this study, a PRDX6 overexpression rat model was constructed through chronic viral plasmids and Adriamycin was used to induce myocardial injury. The serum CK$\mathrm{MB}, \mathrm{Mb}$, and $\mathrm{cTnI}$ levels of Adriamycin rats were found to be significantly higher than those of the control rats, while the serum levels of CK-MB, Mb, and cTnI in
Adriamycin + PRDX6 rats were significantly lower than those in Adriamycin + LV rats. Myocardial tissue is rich in CK-MB, which is currently recognized as an important indicator of myocardial injury and infarction (15). cTnI is primarily distributed in the striated muscle and the polypeptide chain within the submicron structure of myocardial cells. Following damage to myocardial tissue, cTnI can quickly enter the blood system, causing the cTnI content in the blood to increase; therefore, the level changes of $\mathrm{cTnI}$ can reflect the degree of myocardial damage (16). Mb is a small molecule pigment-protein 

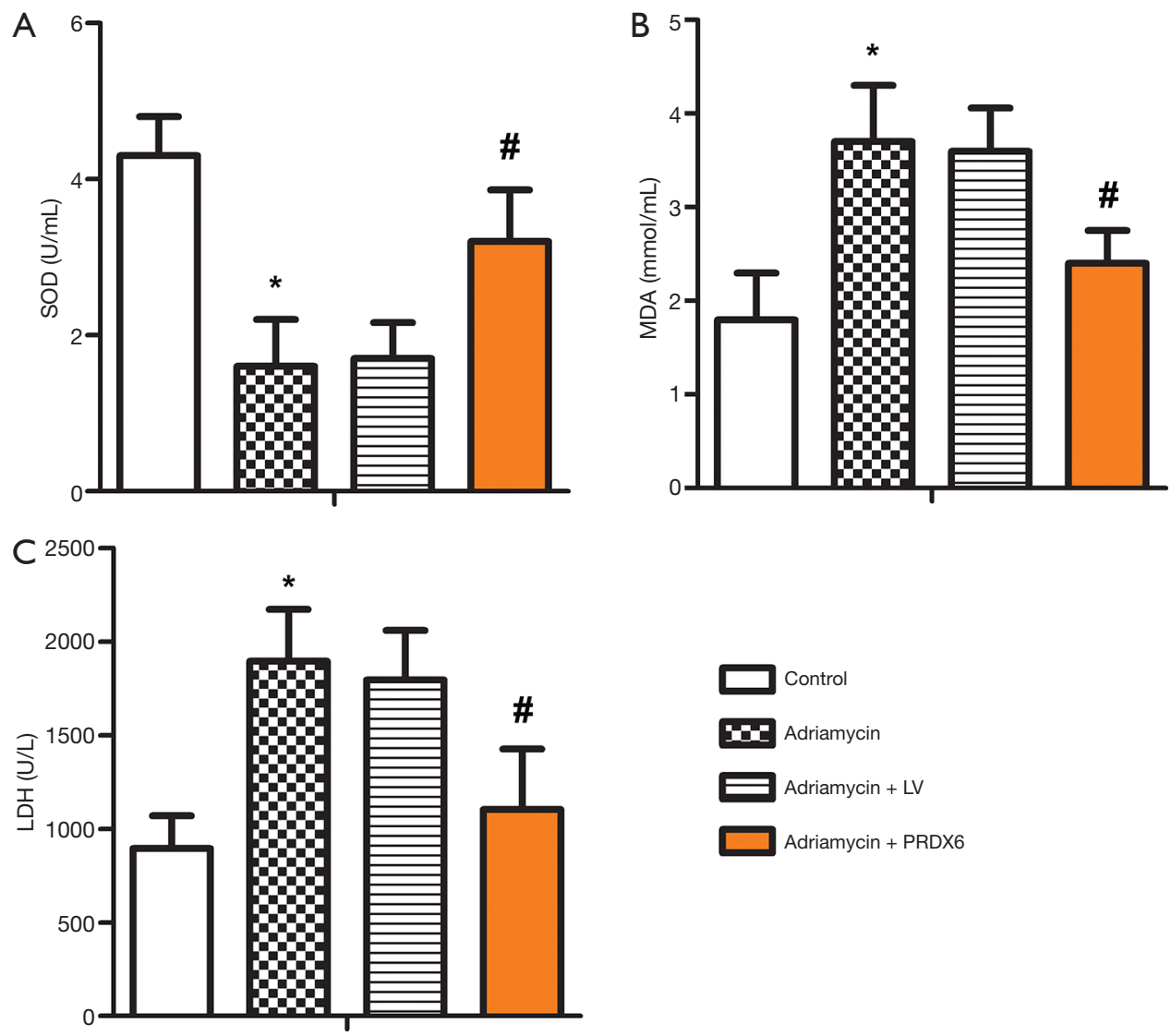

Figure 4 The effects of PRDX6 overexpression on the levels of myocardial oxidative stress markers. (A) Histogram of SOD activity in myocardial tissue from each group of rats; (B) histogram of the level of MDA in myocardial tissue from each group of rats; (C) histogram of the level of $\mathrm{LDH}$ in myocardial tissue from each group of rats. * $\mathrm{P}<0.05$ compared with control group; ${ }^{*}, \mathrm{P}<0.05$ compared with Adriamycin $+\mathrm{LV}$ group.

formed by the combination of globin and methemoglobin that is mainly distributed in the myocardium and striated muscle cells and has the function of transporting and storing oxygen in muscle cells (17). When cardiomyocytes are damaged, $\mathrm{Mb}$ carries out blood circulation from the cardiomyocytes through diffusion. In this study, the changes of myocardial tissue in rats with myocardial injury were observed using HE staining. The myocardial tissue cells of the control and Adriamycin + PRDX6 rats were found to be neatly arranged, without cell necrosis and myocardial fiber rupture, while the myocardial tissue cells of the Adriamycin and Adriamycin $+\mathrm{LV}$ rats were disordered, with severe cell necrosis and broken myocardial fibers. These observations show that doxorubicin can cause myocardial damage in rats, but PRDX6 overexpression can alleviate the severity of this damage.

Adriamycin can cause myocardial tissue damage, which may be attributable to the apoptosis of myocardial cells. This study found that the positive expression rate of caspase- 3 and the levels of Bax/Bcl-2 in the myocardial tissue of the Adriamycin group were significantly higher than those of the control group, while the positive expression rate of caspase- 3 and the level of $\mathrm{Bax} / \mathrm{Bcl}-2$ in the Adriamycin + PRDX6 rats were significantly lower than those in the Adriamycin $+\mathrm{LV}$ rats. The caspase family is a group of proteases that are mainly distributed in the cytoplasm and have a similar structure. Caspases participate in the growth, differentiation, and apoptosis of eukaryotic cells. Caspase- 3 is a member of this subgroup and acts as an apoptotic executor in the process of apoptosis (18). Bcl-2 can hinder the activation of apoptosis signaling caused by DNA damage. An increase in its expression level not only causes changes in mitochondrial membrane potential but can also inhibit the production of free radicals and 
Page 8 of 10
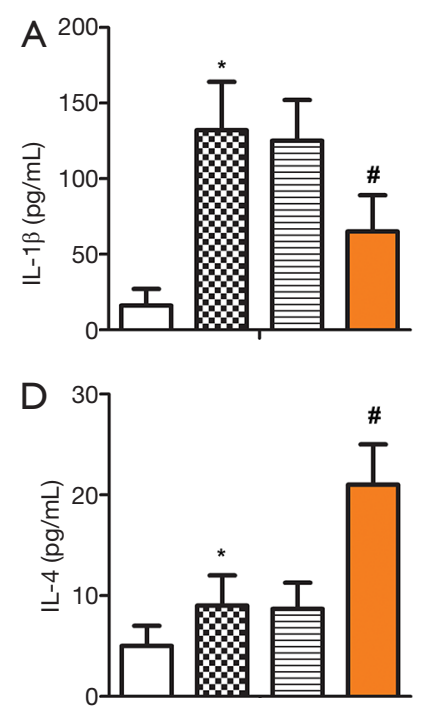
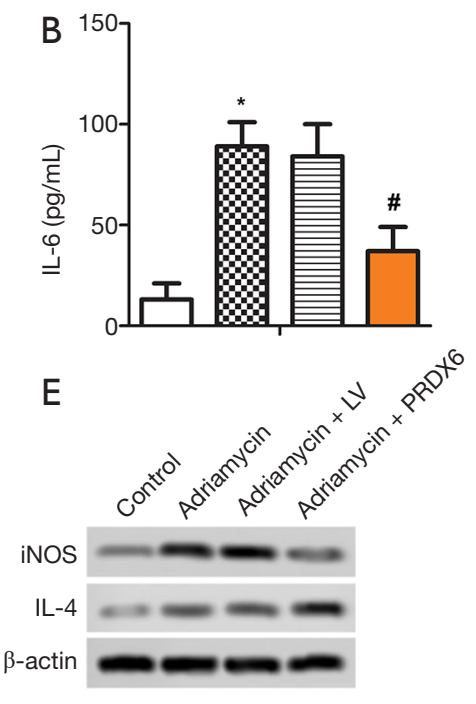

Guo et al. Peroxiredoxin 6 alleviates myocardial injury in rats.
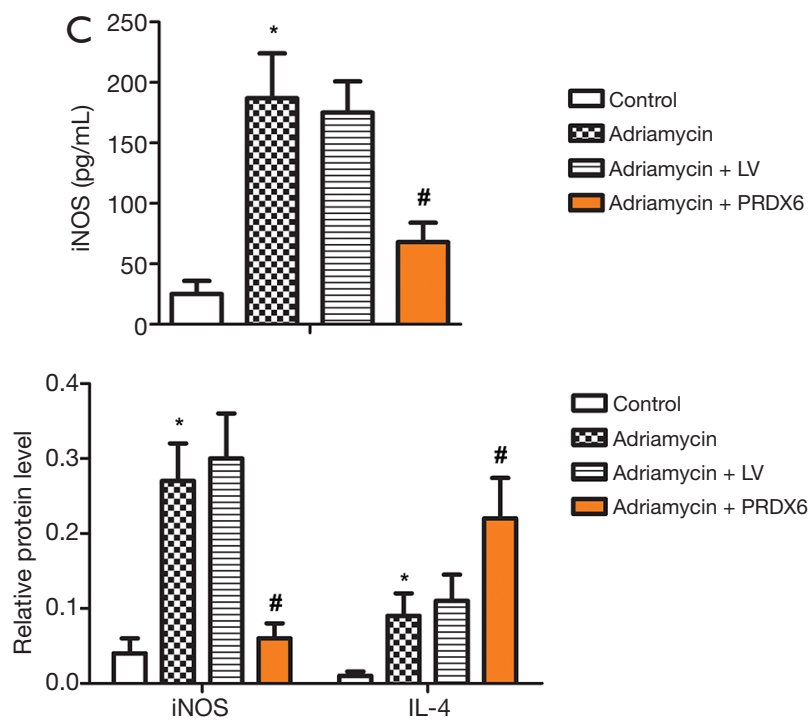

Figure 5 The effects of PRDX6 overexpression on the levels of myocardial inflammatory factors. (A) Histogram of interleukin (IL)-1 $\beta$ levels in the myocardial tissue of each group of rats; (B) histogram of IL-6 levels in the myocardial tissue of each group of rats; (C) histogram of iNOS levels in the myocardial tissue of each group of rats; (D) columnar statistics of IL-4 levels in myocardial tissues from each group of rats; (E) Gel imaging results of the iNOS and IL-4 protein levels in myocardial tissues from each group of rats, and the columnar iNOS and IL-4 protein levels in myocardial tissues from each group of rats. *, $\mathrm{P}<0.05$ compared with control group; ${ }^{*}, \mathrm{P}<0.05$ compared with Adriamycin + LV group.

lipid peroxides to achieve an inhibitory effect on apoptosis $(19,20)$. Bax is a Bcl-2-related protein that enhances the permeability of cell membranes and is able to promote the activation of caspase factor and cell apoptosis. Bax/Bcl-2 can reflect the state of apoptosis $(21,22)$. Hampton et al. (23) reported the potential role of PRDX in the process of cell apoptosis and that it could be used as a marker of oxygen reduction before apoptosis and necrosis. These findings indicate that the overexpression of PRDX6 may reduce the level of Caspases-3 and Bax protein and increase the level of $\mathrm{Bcl}-2$ protein in myocardial tissue, although the mechanism of action is still unclear.

Doxorubicin-induced myocardial damage may be related to the promotion of active oxygen generation in the blood system by doxorubicin, which activates the mitochondrialmediated oxidative stress response in myocardial tissue and induces myocardial damage. The results of this study showed that the levels of MDA and LDH in the myocardial tissue of Adriamycin rats were significantly higher than those in the myocardial tissue of control rats, whereas SOD activity was significantly lower. Meanwhile, the levels of MDA and LDH in the myocardial tissue of Adriamycin + PRDX6 rats were significantly lower than those in the myocardial tissue of Adriamycin + LV rats, while SOD showed the opposite result. MDA is a product of lipid peroxidation, SOD has the function of scavenging oxygen free radicals, and $\mathrm{LDH}$ is a glycolytic enzyme and the level of LDH will increase in myocardium (24). This proves that doxorubicin can improve myocardial oxidative stress response in rats with myocardial injury. PRDX6 can eliminate $\mathrm{H}_{2} \mathrm{O}_{2}$ and peroxide, and has antioxidant effects. PRDX6 overexpression can alleviate the oxidative stress response caused by doxorubicin. However, the mechanism by which PRDX6 overexpression promotes the increase of SOD activity is still unclear.

Apoptosis is closely related to inflammation. The results of this study showed that the levels of IL-1 $\beta$, IL- 6, iNOS, and IL-4, as well as the iNOS and IL-4 protein levels in the myocardial tissue of the Adriamycin rats were significantly higher than those in the myocardial tissue of the control group $(\mathrm{P}<0.05)$. Meanwhile, the levels of IL-1 $\beta$, IL-6, iNOS, and iNOS protein in the myocardial tissue of the Adriamycin + PRDX6 rats were significantly lower than those in the myocardial tissue of the Adriamycin $+\mathrm{LV}$ rats $(\mathrm{P}<0.05)$, while the IL-4 and IL-4 protein levels showed the opposite result $(\mathrm{P}<0.05)$. IL- $1 \beta$ and IL- 6 are pro-inflammatory factors. When IL- $1 \beta$ is induced by activating factors, it activates the 
NF-kB signaling pathway, promotes the secretion and synthesis of IL-6 and other pro-inflammatory factors, and participates in the occurrence of many diseases (25). When the body is stimulated by inflammation, endothelial cells, monocytes, macrophages, and lymphocytes secrete IL-6. IL-4 is an anti-inflammatory factor secreted by T helper 2 (Th2) cells, which promotes the proliferation of $\mathrm{B}$ cells and $\mathrm{T}$ cells as well as the differentiation of CD4+ T cells into Th2 cells, and participates in humoral immunity and adaptive immunity (26). Nitric oxide (NO) is a molecular signal that regulates cardiovascular functions and has an inhibitory effect on leukocyte adhesion, platelet aggregation, and the proliferation of smooth muscle cells. When myocardial tissue is damaged by ischemia and hypoxia, iNOS expression is induced to synthesize NO and regulate myocardial damage (27). This shows that PRDX6 overexpression can improve the inflammatory response of myocardial tissue induced by doxorubicin, although the mechanism of action needs further study.

In conclusion, PRDX6 overexpression can alleviate doxorubicin-induced myocardial injury, myocardial tissue apoptosis, oxidative stress, and inflammatory response in rats. However, the molecular mechanism underlying this effect needs to be studied in depth.

\section{Acknowledgments}

Funding: None.

\section{Footnote}

Reporting Checklist: The authors have completed the ARRIVE reporting checklist. Available at http://dx.doi. org/10.21037/atm-20-6598

Data Sharing Statement: Available at http://dx.doi. org/10.21037/atm-20-6598

Conflicts of Interest: All authors have completed the ICMJE uniform disclosure form (available at http://dx.doi. org/10.21037/atm-20-6598). The authors have no conflicts of interest to declare.

Ethical Statement: The authors are accountable for all aspects of the work in ensuring that questions related to the accuracy or integrity of any part of the work are appropriately investigated and resolved. All experiments are conducted according to the $3 \mathrm{R}$ principle for animal experiments and approved by the ethics committee.

Open Access Statement: This is an Open Access article distributed in accordance with the Creative Commons Attribution-NonCommercial-NoDerivs 4.0 International License (CC BY-NC-ND 4.0), which permits the noncommercial replication and distribution of the article with the strict proviso that no changes or edits are made and the original work is properly cited (including links to both the formal publication through the relevant DOI and the license). See: https://creativecommons.org/licenses/by-nc-nd/4.0/.

\section{References}

1. Hardaway BW. Adriamycin-associated cardiomyopathy: where are we now? updates in pathophysiology, dose recommendations, prognosis, and outcomes. Curr Opin Cardiol 2019;34:289-95.

2. Fan X, Zhong HJ, Zhao BB, et al. Metformin prolonged the survival of diffuse large B-cell lymphoma and grade $3 \mathrm{~b}$ follicular lymphoma patients responding to firstline treatment with rituximab plus cyclophosphamide, doxorubicin, vincristine, and prednisone: a prospective phase II clinical trial. Transl Cancer Res 2018;7:1044-53.

3. Mei S, Hong L, Cai X, et al. Oxidative stress injury in doxorubicin-induced cardiotoxicity. Toxicol Lett 2019;307:41-8.

4. Al-Taee H, Azimullah S, Meeran MFN, et al. $\beta$-caryophyllene, a dietary phytocannabinoid attenuates oxidative stress, inflammation, apoptosis and prevents structural alterations of the myocardium against doxorubicin-induced acute cardiotoxicity in rats: An in vitro and in vivo study. Eur J Pharmacol 2019;858:172467.

5. Rhee SG, Kil IS. Multiple Functions and Regulation of Mammalian Peroxiredoxins. Annu Rev Biochem 2017;86:749-75.

6. Ismail T, Kim Y, Lee H, et al. Interplay Between Mitochondrial Peroxiredoxins and ROS in Cancer Development and Progression. Int J Mol Sci 2019;20:4407.

7. Arevalo JA, Vázquez-Medina JP. The Role of Peroxiredoxin 6 in Cell Signaling. Antioxidants (Basel) 2018;7:172.

8. Yoo YC, Yoo KJ, Lim BJ, et al. Propofol attenuates renal ischemia-reperfusion injury aggravated by hyperglycemia. J Surg Res 2013;183:783-91.

9. Shi H, Yu HJ, Wang HY, et al. Topical administration of peroxiredoxin- 6 on the cornea suppresses inflammation and neovascularization induced by ultraviolet radiation. 
Invest Ophthalmol Vis Sci 2012;53:8016-28.

10. Yang D, Jin M, Bai C, et al. Peroxiredoxin 6 suppresses Muc5ac overproduction in LPS-induced airway inflammation through H2O2-EGFR-MAPK signaling pathway. Respir Physiol Neurobiol 2017;236:84-90.

11. Sharma A, Verma S. Mechanisms by Which GlucagonLike-Peptide-1 Receptor Agonists and Sodium-Glucose Cotransporter-2 Inhibitors Reduce Cardiovascular Risk in Adults With Type 2 Diabetes Mellitus. Can J Diabetes 2020;44:93-102.

12. Cagel M, Grotz E, Bernabeu E, et al. Doxorubicin: nanotechnological overviews from bench to bedside. Drug Discov Today 2017;22:270-81.

13. Thamlikitkul L, Srimuninnimit V, Akewanlop C, et al. Efficacy of ginger for prophylaxis of chemotherapyinduced nausea and vomiting in breast cancer patients receiving adriamycin-cyclophosphamide regimen: a randomized, double-blind, placebo-controlled, crossover study. Support Care Cancer 2017;25:459-64.

14. Songbo M, Lang H, Xinyong C, et al. Oxidative stress injury in doxorubicin-induced cardiotoxicity. Toxicol Lett 2019;307:41-8.

15. FAN J, MA J, XIA N, et al. Clinical Value of Combined Detection of CK-MB, MYO, cTnI and Plasma NTproBNP in Diagnosis of Acute Myocardial Infarction [J]. Clin Lab 2017;63:427-33.

16. Mair J, Lindahl B, Hammarsten O, et al. How is cardiac troponin released from injured myocardium? Eur Heart J Acute Cardiovasc Care 2018;7:553-60.

17. Lam FC, Khan TM, Faidah H, et al. Effectiveness of whey protein supplements on the serum levels of amino acid, creatinine kinase and myoglobin of athletes: a systematic review and meta-analysis. Syst Rev 2019;8:130.

Cite this article as: Guo J, Cao W, Chen C, Chen X. Peroxiredoxin 6 overexpression regulates adriamycin-induced myocardial injury, oxidative stress and immune response in rats. Ann Transl Med 2020;8(20):1320. doi: 10.21037/atm-20-6598
18. Lu W, Lu T, Wei X. Downregulation of DNMT3a expression increases miR-182-induced apoptosis of ovarian cancer through caspase-3 and caspase-9-mediated apoptosis and DNA damage response. Oncol Rep 2016;36:3597-604.

19. Knight T, Luedtke D, Edwards H, et al. A delicate balance-The BCL-2 family and its role in apoptosis, oncogenesis, and cancer therapeutics. Biochem Pharmacol 2019;162:250-61.

20. Ashkenazi A, Fairbrother WJ, Leverson JD, et al. From basic apoptosis discoveries to advanced selective BCL-2 family inhibitors. Nat Rev Drug Discov 2017;16:273-84.

21. Jezek J, Chang KT, Joshi AM, et al. Mitochondrial translocation of cyclin $\mathrm{C}$ stimulates intrinsic apoptosis through Bax recruitment. EMBO Rep 2019;20:e47425.

22. Maes ME, Schlamp CL, Nickells RW. Live-cell imaging to measure BAX recruitment kinetics to mitochondria during apoptosis. PLoS One 2017;12:e0184434.

23. Hampton MB, O'Connor KM. Peroxiredoxins and the Regulation of Cell Death. Mol Cells 2016;39:72-6.

24. Islam MT. Oxidative stress and mitochondrial dysfunctionlinked neurodegenerative disorders. Neurol Res 2017;39:73-82.

25. Theoharides TC, Tsilioni I, Patel AB, et al. Atopic diseases and inflammation of the brain in the pathogenesis of autism spectrum disorders. Transl Psychiatry 2016;6:e844.

26. Gour N, Wills-Karp M. IL-4 and IL-13 signaling in allergic airway disease. Cytokine 2015;75:68-78.

27. Lee M, Rey K, Besler K, et al. Immunobiology of Nitric Oxide and Regulation of Inducible Nitric Oxide Synthase. Results Probl Cell Differ 2017;62:181-207.

(English Language Editor: J. Reynolds) 